\title{
Genetic aberrations of c-myc and CCND/ in the development of invasive bladder cancer
}

\author{
AD Watters', Z Latif ', A Forsyth', I Dunn'2, MA Underwood', KM Grigor ${ }^{3}$ and JMS Bartlett*,I \\ 'University Department of Surgery, Level II, Queen Elizabeth Building, Glasgow Royal Infirmary, Glasgow G3I 2ER, UK; ' ${ }^{2}$ Department of Surgery, Southern \\ General Hospital, Glasgow, UK; ${ }^{3}$ University Department of Pathology, Western General Hospital, Edinburgh EH4 2XU, UK
}

Detrusor muscle invasive transitional cell carcinoma is associated with poor prognosis and is responsible for the majority of bladder cancer related deaths. Amplifications of c-myc and CCND/ are associated with detrusor-muscle-invasive transitional cell carcinoma, however, their precise role in driving disease progression is unclear. Fluorescence in situ hybridisation on archival tissue from 16 patients with primary diagnosis of $\geqslant p T 2$ transitional cell carcinoma and 15 cases with primary $\mathrm{pTa} / \mathrm{p} T \mathrm{I}$ disease subsequently progressing to detrusor-muscle-invasion was performed, in the latter group both pre and post muscle invasive events were studied. No patients presenting with $\geqslant p T 2$ had amplification of c-myc, two out of 16 (I2.5\%) had CCNDI amplification. Of patients who developed $\geqslant p T 2$, two out of 15 (13.3\%) had amplification of c-myc, both in $\geqslant p T 2$, five out of $15(33.3 \%)$ had CCND I amplification, two in pTa/pTI tumours, three in $\geqslant p T 2$ transitional cell carcinomas. In total, two out of 31 (6.5\%) of patients' $\geqslant$ PT2 TCCs were amplified for c-myc and six out of 31 (19\%) were amplified for CCNDI. Eighty-seven per cent (40 out of 46) of tumours were polysomic for chromosome 8 and $80 \%$ (37 out of 46) were polysomic for chromosome II and this reflected the high copy numbers of c-myc and CCND I observed. In almost all cases an increase in c-myc/CCND / copy number occurred prior to invasion and persisted in advanced disease. Amplification of CCND/ or alterations in c-myc/CCND/ early in bladder cancer may have clinical relevance in promoting and predicting progression to detrusor-muscle-invasive transitional cell carcinoma.

British Journal of Cancer (2002) 87, 654-658. doi:I0.1038/sj.bjc.660053I www.bjcancer.com

(c) 2002 Cancer Research UK

Keywords: transitional cell carcinoma; oncogenes; prognosis; c-myc; CCND I

Muscle invasive transitional cell carcinoma (TCC) of the urinary bladder (characterised by invasion to the detrusor-muscle wall of the bladder or beyond) has a poor prognosis. Invasive TCC is routinely treated with radiotherapy or surgery but, despite treatment, up to $50 \%$ of patients develop metastases and die within 5 years (reviewed by van der Meijden, 1998). A large number of studies have attempted to identify which molecular markers may be driving progression, ultimately to develop targeted therapies (reviewed by Unwin et al, 1999; Reznikoff et al, 2000; Witjes et $a l, 2000)$. Cell cycle regulators such as p53 and Rb have been implicated in bladder cancer progression (reviewed by Knowles, 1999).

Previous research within this laboratory demonstrated that polysomy of chromosomes 9, 7 and 17 are associated with recurrence (Bartlett et al, 1998, Watters et al, 2000), but that these genetic events are not linked with polyploidy (Bartlett et al, 1999). A small study within this laboratory investigating the drug metabolising enzyme NAT2 (the gene is located at chromosome 8p22) in TCC recurrence or progression suggested that polysomy 8 was associated with disease progression (Watters et al, 2001). Further research showed an association with polysomy of one or eight in preinvasive TCCs associated with subsequent invasion (Watters et al, 2002).

Gene amplification has been postulated as a mechanism driving progression in many carcinomas. c- $m y c$, located at $8 \mathrm{q} 24$, encodes for a nuclear transcription factor that regulates cell proliferation

*Correspondence: JMS Bartlett; E-mail: j.m.bartlett@clinmed.gla.ac.uk Received I2 February 2002; revised 20 June 2002; accepted 25 June 2002 and differentiation (Visscher et al, 1997). Gene amplification or increased copy number of $c-m y c$ has been reported in breast (Visscher et al, 1997), head and neck and ovarian cancer (Sauter et al, 1995). There has been a suggestion that gain of chromosome $8 \mathrm{q}$ is involved in progression in bladder cancer (Sauter et al, 1995). 8q24 gains have been associated with advanced stage and grade bladder cancer (Wagner et al, 1997). Low rates of amplification $(15 \%)$ at the $11 \mathrm{q} 13$ region have been reported in bladder cancer (reviewed by Schuuring, 1995). CCND1 maps to 11q13 (Bringuier et al, 1996), involved in the regulation of the G1/S phase transition of the cell cycle (Bringuier et al, 1996).

Following on from previous studies within this laboratory (Bartlett et al, 1998, 1999; Watters et al, 2000, 2001) which associated specific chromosomal aberrations with recurrence or progression, we hypothesised that alteration of key cell cycle modulators $\mathrm{c}-m y c$ and $C C N D 1$ via gene alterations drives progression of superficial and locally invasive TCC (pTa/pT1). This study determined chromosome and gene copy number by FISH in a cohort of patients with either detrusor muscle invasive TCC at presentation or who developed detrusor muscle invasive TCC following a presentation with non-muscle invasive ( $\mathrm{pTa} / \mathrm{pT} 1)$ TCC.

\section{MATERIALS AND METHODS}

\section{Patients}

Sixteen patients with $\geqslant \mathrm{pT} 2$ disease and seven with pTa and eight with $\mathrm{pT} 1$ disease that progressed to $\geqslant \mathrm{pT} 2$ were identified 
from a bladder cancer database in the Department of Surgery, Glasgow Royal Infirmary. Patients were categorised into two groups: PP (progressed at presentation; patients with $\geqslant \mathrm{pT} 2$ at initial diagnosis) and RP (recurrent and progressive; patients with $\mathrm{pTa} / \mathrm{pT} 1$ disease at initial diagnosis who subsequently recurred and progressed with their disease). In order to attempt to understand the genetic events underlying progression from pTa or pT1 TCC, the pre and post muscle-events were studied in the RP group; the presenting lesion in the $\mathrm{PP}$ group. This approach was based on previous research within this laboratory that demonstrated that there was a considerable increase in genetic aberrations from patients with a primary pTa or pT1 TCC that progressed to detrusor-muscle-invasion when compared to those with non-invasive recurrences (Watters et al, 2002).

A total of 46 tumours were studied. Five micron formalin fixed paraffin processed tissue sections of each tumour were cut onto sialinised slides and baked at $56^{\circ} \mathrm{C}$ overnight. All TCCs analysed had a representative section stained with haematoxylin and eosin and were restaged and regraded by a specialist urological pathologist (KMG) following current UICC guidelines (Sobin and Wittekind, 1995).

\section{Fluorescence in situ hybridisation (FISH)}

To determine gene and chromosome copy numbers, levels of gene amplification or deletion in situ, FISH, using locus specific DNA probes for c-myc, CCND1 and chromosome enumeration probes for eight and 11 in a dual hybridisation reaction, was applied to five micron tissue sections of tumour. Included in each run was bladder tissue with normal copy number for all the probes used.

The FISH methodology was followed as outlined: Tissue sections were dewaxed and rehydrated, then subjected to pretreatments $0.2 \mathrm{~N} \mathrm{HCl}$ for $20 \mathrm{~min}$ at room temperature, $8 \%$ sodium thiosulphate at $80^{\circ} \mathrm{C}$ for $30 \mathrm{~min}$ and $0.5 \%$ pepsin in $0.2 \mathrm{~N} \mathrm{HCl}$ for $26 \mathrm{~min}$ at $37^{\circ} \mathrm{C}$. Tissue sections were post-fixed in $10 \%$ neutral buffered formalin at room temperature for $10 \mathrm{~min}$ before dehydration in ascending grades of alcohol and air drying. These steps were carried out on a VP2000 robotic pretreatment slide processor (Vysis, UK, Ltd).

The tissue sections were assessed for extent of digestion as previously outlined (Watters et al, 2000). Tissue sections were denatured in $70 \%$ formamide, $2 \times \mathrm{SSC} \mathrm{pH} 7-8$ at $72^{\circ} \mathrm{C}$ for $5 \mathrm{~min}$ on the Omnislide hybridisation module (Hybaid, UK Ltd), dehydrated and air-dried. Two separate experiments were carried out on adjacent sections, with probes for the pericentromere of chromosome 8, labelled with SpectrumGreen ${ }^{\mathrm{TM}}$ and a SpectrumOrange ${ }^{\mathrm{TM}}$ labelled probe for $\mathrm{c}-m y c$ (Vysis, UK). The alternative probes set was probes for the pericentromere of chromosome 11, labelled with SpectrumGreen ${ }^{\mathrm{TM}}$ and a SpectrumOrange $^{\mathrm{TM}}$ labelled probe for CCND1 (Vysis, UK). Both combinations were applied as a double label. For each section, $1 \mu \mathrm{l}$ of each probe was added to $7 \mu$ l hybridisation $\operatorname{mix}(50 \%$ formamide, $2 \times$ SSC, $10 \%$ dextran sulphate) and $1 \mu$ l deionised water and denatured in a waterbath at $72^{\circ} \mathrm{C}$ for $5 \mathrm{~min}$, applied to the tissue sections and hybridised overnight at $37^{\circ} \mathrm{C}$ (Vysis, Inc).

Following posthybridisation washes in $0.4 \times$ SSC, $0.3 \%$ Nonidet $30, \mathrm{pH} 7$ at $72^{\circ} \mathrm{C}$ for $2 \mathrm{~min}$, the tissue sections were mounted in $0.25 \mu \mathrm{g} \mathrm{ml}^{-1}$ DAPI in antifade (Vectashield, UK) and viewed with a Leica DMLB microscope. A triple band pass filter block spanning the excitation and emission wavelengths of the SpectrumGreen $^{\mathrm{TM}}$ and SpectrumOrange ${ }^{\mathrm{TM}}$ fluors (Vysis, UK) and DAPI was used in the analysis of the hybridisation. Image capture was achieved using a digital camera and software (Leica DC200, Leica UK).

\section{Scoring criteria}

Serially sectioned haematoxylin and eosin stained tissue sections were first examined microscopically to localise areas of TCC. FISH stained sections were then scanned at $\times 400$ magnification to localise the TCC areas identified. Three distinct areas were identified and signals per nucleus in 20 nuclei per area for chromosome 8 and $\mathrm{c}-m y c$ and chromosome 11 and CCND1 respectively were counted at $\times 1000$ magnification and the results recorded manually. Average chromosome and gene copy number per area was calculated by totalling the number of signals counted and dividing this figure by the number of nuclei assessed. Mean copy number per section was calculated by combining the results for the three areas. Where chromosomal heterogeneity was noted the most abnormal score was used in the final analysis. Control sections from normal bladder tissue obtained from post mortem normal bladder were included in the analysis. Values for disomy were derived from the analysis of these tissue sections as previously reported (Bartlett et al, 1998; Watters et al, 2000).

The ratio of gene to chromosome copy number was determined by dividing the number of signals for $c-m y c$ or CCND1 by the number of signals for chromosome 8 or 11 respectively. Gene amplification was defined as a ratio greater than two, based on values determined by Vysis Inc and incorporated in their guidelines for using the PathVysion ${ }^{\text {TM }}$ system to determine amplification levels of the HER2/neu gene in breast cancer in a diagnostic setting (Bartlett et al, 2001). This was based on the normal mean copy number for the gene as being less than four signals per nucleus. In some cases with high amplification ratios ( $>5$ copies per chromosome), clusters of gene signals, analogous to homogeneous staining regions, were observed. In these cases it was not possible to accurately assess the true gene copy number, but by focusing through the cluster a reasonable estimate was achieved. In none of these cases did the presence of a cluster of signals alter the final result.

\section{Statistics}

Crosstabs (SPSS for windows release 9.0) was used to compare similarity of copy number between chromosomes 8 and 11 and c-myc and CCND1.

\section{RESULTS}

\section{Patients}

The average age of the PP group was 70.9 years, (range 50 to 89 years) and the RP group 68.9 years (range 43 to 84 years). The $\mathrm{M}: \mathrm{F}$ ratio was $13: 3$ in the PP group, $12: 3$ in the RP group. Mean follow-up for the PP group was 42 months (range 6-91) and for the RP group 39 months (range $3-117$ ). In the PP group, 13 patients were treated with radiotherapy, one died before radiotherapy commenced, one refused radiotherapy and one had a cystectomy. In the RP group, six had radiotherapy after the muscle-invasive event, five had a cystectomy, two had no treatment. One patient had palliative radiotherapy early in his disease history, subsequently recurred with pT1 and pTa TCCs and eventually, 13 years later, succumbed to his disease, and one patient had radiotherapy before his muscle-invasive TCC. Of the PP group, 11 patients died from their disease, range 6 to 59 months following diagnosis. The four patients still alive had no evidence of local or metastatic disease at last clinic appointment. Of the RP group 9 patients died of their disease, range 3-15 months following diagnosis of invasive disease. Of the six patients still alive, two had no evidence of local or metastatic disease at last clinic appointment, two had metastatic disease, and there were no records following diagnosis with invasive TCC for the remaining two. 


\section{FISH}

All hybridisations were successful apart from that of one PP with the probes for $c-m y c$ and chromosome 8 . The normal range for copy number of each DNA probe is detailed in Table 1, and an example of a TCC with disomy of $c-m y c$ and chromosome 8 is shown in Figure 1A.

\section{c-myc and chromosome 8}

Fourteen out of 15 (93\%) PP tumours had increased copy number of c-myc and chromosome 8, between 2.15 and 6.35 for c-myc and between 2.03 and 7.73 for chromosome 8 (Figure 1B). None of the tumours exhibited gene amplification (Table 2).

Twenty-seven out of 30 (90\%) RP tumours had increased copy number for $c-m y c$ of between 2.03 and 5.53. Of these 14 out of $15(93 \%)$ were abnormal in the pTa and pT1 TCCs, the TCC was pTa and 13 out of $15(87 \%)$ were abnormal in the $\geqslant$ pT2 TCC (Table 2). Twenty-seven out of 30 (90\%) RP tumours had polysomy 8 , (range 2.05 to 4.89 ). Of these 13 out of $15(87 \%)$ were polysomic in the pTa or pT1 TCCs, both normal TCCs were pTa and 14 out of $15(93 \%)$ were polysomic in the pT2+ TCC. Two out of $30(6.7 \%)$ tumours had gene amplification, and both were $\geqslant \mathrm{pT} 2$.

There was a close correlation between c-myc and chromosome 8 copy number, $P=0.001$.

\section{CCND1 and chromosome 11}

Fourteen out of $16(87.5 \%)$ PP tumours had an increase of CCND1, copy number between 2.70 and 13.18 (Table 2) and 12 out of $16(75 \%)$ had polysomy 11 , copy number between 2.70 and 5.88 (Figure 1C). Of these, two (12.5\%) had amplification of CCND1 (Table 2, Figure 1D).

Twenty-four out of $30(80 \%)$ RP tumours had an increase in CCND1 (range 2.11 to 9.00 , Table 2). Of these 12 out of 15 $(80 \%)$ were abnormal in the pTa or pT1 TCCs; two of the normal tumours were pTa, one was pT1, and 12 out of $15(80 \%)$ were abnormal in the pT2+ TCC. Twenty-three out of $30(77 \%)$ RP tumours were polysomic for chromosome 11, copy number between 2.13 and 4.57 . Of these 11 out of $15(73 \%)$ were polysomic in the pTa or pT1 TCC; three of the normal TCCs were $\mathrm{pTa}$, one was pT1, and 12 out of $15(80 \%)$ were polysomic in the pT2+ TCC. Six out of $30(20 \%)$ tumours had amplification of CCND1, four of which were pT2 or above, the remaining two were pT1G3 and pTaG3. Of these one patient had amplification in their pre and post muscle invasive tumours. There was a close correlation with CCND1 and chromosome 11 copy number, $P<0.001$.

In total six out of 31 patients $(19 \%)$ had amplification of CCND1 in their detrusor muscle invasive tumours, compared to two out of $31(6.5 \%)$ patients with amplification of $c-m y c, \mathrm{~N} / \mathrm{S}$. Both patients with amplification of $c-m y c$ had amplification of CCND1.

Data from the results of the assessment of gene copy number in the non-muscle invasive (pTa or pT1) TCCs is depicted in Table 3. This shows similar rates of increased copy number in $\mathrm{pTa}$ or $\mathrm{pT} 1$ tumours.

\section{DISCUSSION}

The observation that amplification of oncogenes predominates in later stages of cancer progression (Ried et al, 1999; Reznikoff et $a l, 2000)$ and that the majority of genetic alterations in TCC occur prior to progression (Tsao et al, 2000) implies that genetic instability of this type may influence progression. The aim of this study was to determine if changes of the oncogenes c-myc and CCND1 in preinvasive tumours drive later progression. Abnormal regula-
Table I Normal values for chromosome and gene copy number

\begin{tabular}{lcc}
\hline Probe & Copy number & Normal range \\
\hline c-myc & $1.70 \pm 0.03$ & 1.61 to 1.79 \\
Chromosome 8 & $1.76 \pm 0.08$ & 1.52 to 2.00 \\
CCNDI & $1.74 \pm 0.11$ & 1.41 to 2.07 \\
Chromosome |I & $1.72 \pm 0.13$ & 1.33 to 2.11 \\
\hline
\end{tabular}

Values for normal copy number were first established by evaluating normal bladder tissue. The normal range for disomy was defined as the mean $\pm 3 \times$ s.d. $(99 \% \mathrm{Cl}$ )

tion of c-myc can result in phenotypic transformation, aberrant cell cycle control and genomic instability. Disruption of CCND1, that encodes for cyclin D1, involved in the regulation of the G1/ $S$ transition of the cell cycle (Bringuier et al, 1996), may form the basis of genetic instability (Reznikoff et al, 2000).

The small numbers of tumours exhibiting gene amplification of c-myc observed here is in accordance with previous studies (Sauter et al, 1995). However, rates of amplification of CCND1 were $19 \%$, marginally higher than a previous study of bladder cancer by Southern blotting which reported amplification rates of $11 \mathrm{q} 13$ of $11 \%$ (Bringuier et al, 1996). The candidate oncogenes in the region $11 \mathrm{q} 13$ in bladder cancer postulated by Bringuier et al (1996) are CCND1 and EMS1 (a gene involved in regulating the interactions between components in the adherens junctions, Schuuring (1995)).

Both the 8q24 (that contains $c-m y c$ ) and $11 \mathrm{q} 13$ regions are frequently involved in translocations in lymphomas (Arber, 2000). Burkitt's lymphoma is usually associated with a translocation of c-myc and CCND1 has been suggested as a marker for mantle cell lymphoma (Arber, 2000). Although translocations are not uncommon in bladder cancer, translocation as a sole cytogenetic event has not been described (Sandberg and Berger, 1994). However, the chromosomal regions that contain c-myc and CCND1 are genetically unstable, which may be of significance in the development of muscle-invasive bladder cancer.

Previously, research from this laboratory has shown an observed increase in aneusomy from pTa to pT1 TCCs, but a lack of association with stage and recurrence or progression, suggesting that it is aneusomy and not pathological classification which is driving progression in pTa or pT1 tumours (Watters et al, 2001, 2002; Edwards et al, 2001). Furthermore, it is of interest to note that the rate of genetic abnormalities is similar when comparing the data from patients who progress from nonmuscle-invasion and those who present with muscle invasion. In this study, and previously, we have shown that there are higher rates of aneusomy in patients who progress to muscle invasion compared to those who recur with $\mathrm{pTa} / \mathrm{pT} 1$ disease (Watters et al, 2000, 2001, 2002). Muscle-invasive TCCs are characterised by a large number of cytogenetic alterations including overrepresentation of $8 \mathrm{q}$ and $11 \mathrm{q} 13$ (Tomovska et al, 2001). Thus this study would support the finding by Tsao et al (2000) that the majority of genetic alterations in TCC occur prior to progression. They showed that there was an increase of only $10 \%$ of genetic aberrations in metastases compared to primary cell lines from two patients reported in their study, and they suggest that most allelic losses occur before progression. It is widely recognised that genetic instability increases from precursor, or in the case of TCC, preinvasive lesions, to invasion (Ried et al, 1999). In our study we also observed that there was a very high rate of abnormalities in preinvasive lesions, regardless of stage.

A gain of chromosome $8 \mathrm{q}$ may contribute to bladder cancer progression (Sauter et al, 1995). The gain of a large fragment of $8 \mathrm{q}$ also known as an isochrome $8 \mathrm{q}$ has been observed in bladder 

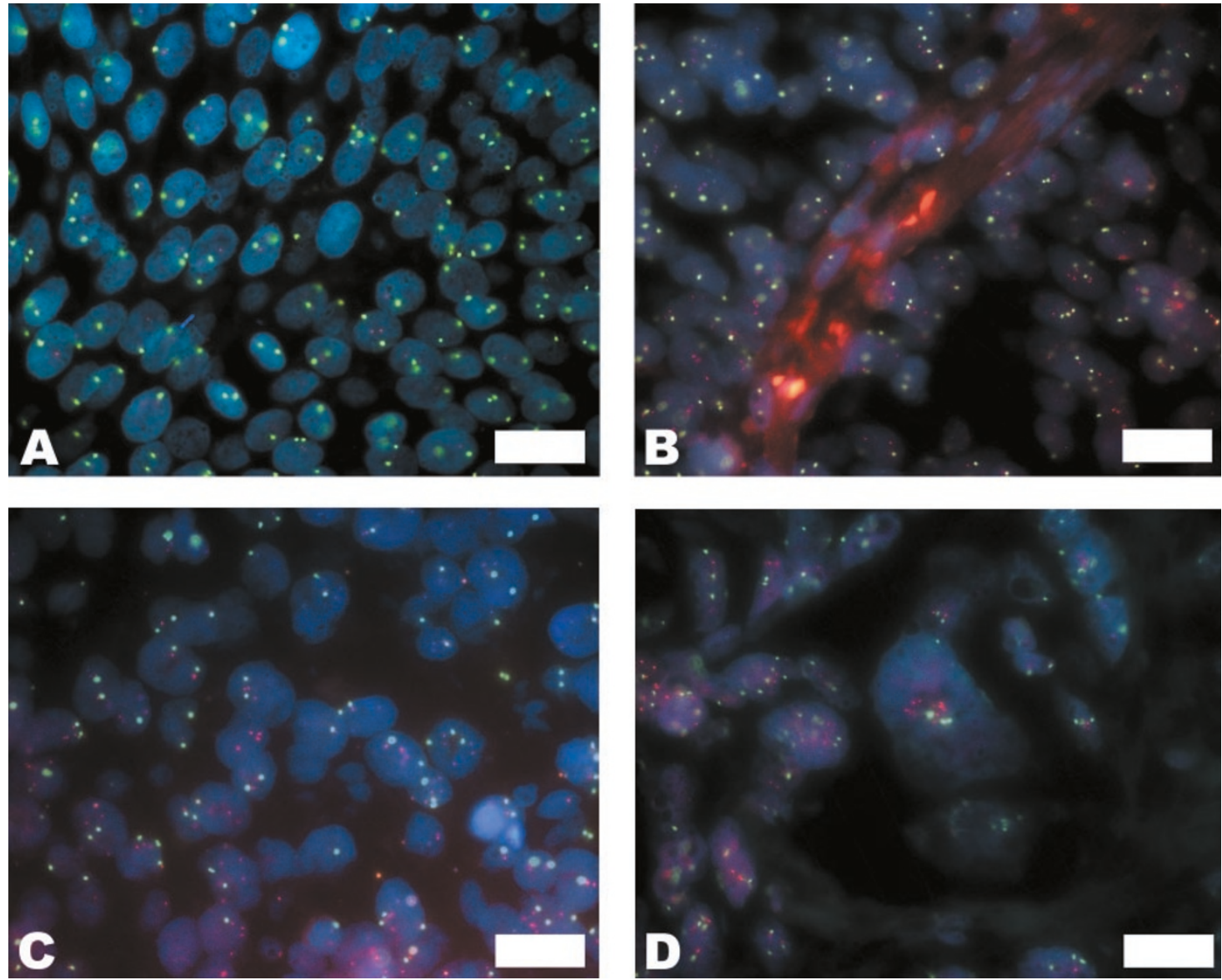

Figure I FISH size bar on all photomicrographs is 20 microns. Chromosome copy depicted in green, gene copy in red, nuclei stained with DAPI, fluorescing blue. (A) Disomy of c-myc and chromosome 8, PP pT2 G3. (B) Polysomy of c-myc and chromosome 8, RP pTIcG3. (C) Polysomy of CCND I and chromosome II, RP, pT2G3. (D) Amplification of CCNDI, RP, PT2G3.

Table 2 Percentage of TCCs with increased gene copy number for c-myc and CCND I

\begin{tabular}{llcccc}
\hline $\begin{array}{l}\text { Patient } \\
\text { group }\end{array}$ & Stage & $\begin{array}{c}\text { Increased c-myc } \\
\text { copy number }\end{array}$ & c-myc amp & $\begin{array}{c}\text { Increased CCNDI } \\
\text { copy number }\end{array}$ & CCNDI amp \\
\hline $\mathrm{PP}$ & $\mathrm{PT2}$ & $14 / 15(93 \%)$ & 0 & $14 / 16(87.5 \%)$ & $2 / 16(12.5 \%)$ \\
$\mathrm{RP}$ & $\mathrm{PTa} / \mathrm{pTI}$ & $14 / 15(93 \%)$ & 0 & $12 / 15(80 \%)$ & $2 / 15(13 \%)$ \\
$\mathrm{RP}$ & $\mathrm{PT}$ & $13 / 15(87 \%)$ & $2 / 15(13 \%)$ & $12 / 15(80 \%)$ & $4 / 15(27 \%)$ \\
\hline
\end{tabular}

$\mathrm{PP}=$ progressed at presentation, patients with $\geqslant \mathrm{pT} 2$ at initial diagnosis; $\mathrm{RP}=$ recurrer progressors, patients with $\mathrm{PTa} / \mathrm{pTI}$ disease at initial diagnosis who subsequently recurred and progressed with their disease; amp=gene amplification. There was a high percentage of tumours with elevated gene copy number but no significant differences between the patient groups. Of those with elevated gene copy number, a small proportion also had gene amplification.

Table 3 Comparison of rates of increased gene copy number and gene amplification between stage $\mathrm{pTa}$ and $\mathrm{pTI}$

\begin{tabular}{lcccc}
\hline Stage & $\begin{array}{c}\text { Increased c-myc } \\
\text { copy number }\end{array}$ & $\begin{array}{c}\text { Amp } \\
\text { c-myc }\end{array}$ & $\begin{array}{c}\text { Polysomy } \\
\text { CCNDI }\end{array}$ & $\begin{array}{c}\text { Increased CCNDI } \\
\text { copy number }\end{array}$ \\
\hline $\mathrm{PTa}$ & $6 / 7$ & 0 & $4 / 7$ & $1 / 15$ \\
$\mathrm{PTI}$ & $8 / 8$ & 0 & $7 / 8$ & $1 / 15$ \\
\hline
\end{tabular}

This table illustrates the rates of anuesomy and amplification in patients with $\mathrm{pTa}$ or PTI TCCs that represented with detrusor-muscle-invasion ( $\geqslant p T 2)$. Rates were similar for either PTa or PTI TCCs. cancer (Sauter et al, 1995). The stronger increase in 8q24 gains from $\mathrm{pT} 1$ to $\mathrm{pT} 2-4$ than from $\mathrm{pTa}$ to $\mathrm{pT} 1$ could suggest that $8 \mathrm{q}$ gains occur later in bladder cancer progression than $8 \mathrm{p}$ deletions (Wagner et al, 1997). In a study of chromosomal aberrations by comparative genomic hybridisation, there was a very small percentage of gains of $11 \mathrm{q} 13$ in pTa tumours. However, in pT1 tumours, loss of chromosome 11 was observed with the exception of 11q13 which was often gained or amplified (Simon et al, 1998). In this study, c-myc and CCND1 may be dysfunctional due to the observed increased copy number, and it is possible that 
protein expression is increased as a consequence which may be a route to muscle-invasion.

When the results from this study were compared to a previous study in this laboratory that examined HER2/neu and chromosome 17 levels in TCC (unpublished data), very similar results were obtained i.e high levels of polysomy and much lower levels of amplification. This suggests that tumours that progress to pT2 and above, or present with that phenotype, have high levels of polysomy of many chromosomes, and that they may exhibit polyploidy. However, we have investigated, by the use of multiple FISH probes, the rate of polysomy in this cohort and have found that this cannot explain the findings in this study (Bartlett et al, 1999). Therefore we conclude that the alterations in these chromosomes represent events which are closely associated with disease progression and independent of polyploidisation. Ried et al (1999) noted that the number of chromosomal copy alterations increased significantly in the transition from precursor lesions to

\section{REFERENCES}

Arber DA (2000) Molecular diagnostic approach to non-Hodgkin's lymphoma. J Mol Diagnostics 2: 178-190

Bartlett JMS, Adie L, Watters AD, Going JJ, Grigor KM (1999) Chromosome aberrations in TCC predictive of disease outcome are independent of polyploidy. Br J Urol 84: 775-779

Bartlett JMS, Going JJ, Mallon EA, Watters AD, Reeves JR, Stanton P, Richmond J, Donald D, Ferrier R, Cooke TG (2001) Evaluating HER2 amplification and overexpression in breast cancer. J Pathol 195: 422-428

Bartlett JMS, Watters AD, Ballantyne SA, Going JJ, Grigor KM, Cooke TG (1998) Is chromosome 9 loss a marker of disease recurrence in transitional cell carcinoma of the urinary bladder? Br J Cancer 77: 2193-2198

Bringuier P, Tamimi Y, Schuuring E, Schalken J (1996) Expression of cyclin D1 and EMS1 in bladder tumours; relationship with chromosome 11q13 amplification. Oncogene 12: $1747-1753$

Edwards J, Duncan P, Going JJ, Watters AD, Grigor KM, Bartlett JMS (2001) Loss of heterozygosity on chromosomes 11 and 17 are markers of recurrence in TCC of the bladder. Br J Cancer 85: 1894-1899

Knowles MA (1999) Identification of novel bladder tumour suppressor genes. Electrophoresis 20: $269-279$

Reznikoff CA, Sarkar S, Jülicher KP, Burger MS, Jairaj AP, Jarrad DF, Newton MA (2000) Genetic alterations and biological pathways in human bladder cancer pathogenesis. Urol Oncol 5: 191-203

Ried T, Heselmeyer-Haddad K, Blegen H, Schrock E, Auer G (1999) Genomic changes defining the genesis, progression, and malignancy potential in solid human tumours: A phenotype/genotype correlation. Genes Chrom Cancer 25: $195-204$

Sandberg AA, Berger CS (1994) Review of chromosome studies in urological tumours. II. Cytogenetics and molecular genetics of bladder cancer. J Urol 51: $545-560$

Sauter G, Carroll P, Moch H, Kallioniemi A, Kerschmann R, Narayan P, Mihatsch MJ, Waldman FM (1995) C-myc copy number gains in bladder cancer detected by fluorescence in situ hybridization. Am J Pathol. 146: $1131-1139$

Schuuring E (1995) The involvement of the chromosome 11q13 region in human malignancies: cyclin D1 and EMS1 are two new candidate oncogenes - a review. Gene 159: 83-96

Simon R, Burger H, Brinkschmidt C, Bocker W, Hertle L, Terpe H-J (1998) Chromosomal aberrations associated with invasion in papillary superficial bladder cancer. J Pathol 185: 345-351 invasive carcinomas, reflecting an overall genetic instability which we have also observed here.

Further studies of gene alterations in non-invasive non progressive patient groups and investigations of protein expression are required to clarify the effects of genetic abnormalities of c-myc and CCND1 on progression of TCC. Nevertheless, this study has added to the growing evidence that genetic alterations prior to detrusor muscle invasion delineate those cancers which will progress and by extension these changes are associated with genes which therefore drive progression.

\section{ACKNOWLEDGEMENTS}

The contribution of DNA probes by Vysis, Inc to carry out this study is gratefully acknowledged.
Sobin LH, Wittekind Ch (1995) TNM Classification of Malignant Tumours, 5th edn, Union Internationale Contre le Cancer: Wiley-Liss

Tomovska S, Richter J, Süess K, Wagner U, Rozenblum E, Gasser TC, Moch H, Mihatsch MJ, Sauter G, Schraml P (2001) Molecular cytogenetic alterations associated with rapid tumour cell proliferation in advanced urinary bladder cancer. Int J Onco 18: 1239-1244

Tsao J-L, Yatabe Y, Markl IDC, Hajyan K, Jones PA, Shibata D (2000) Bladder cancer genotype stability during clinical progression. Genes Chrom Cancer 29: 26 - 32

Unwin RD, Knowles MA, Selby PJ, Banks RE (1999) Urological malignancies and the proteomic-genomic interphase. Electrophoresis 20: $3629-3637$

van der Meijden PAM (1998) Bladder cancer-clinical review. BMJ 317: 1366 1369

Visscher DW, Wallis T, Awussah S, Mohamed A, Crissman JD (1997) Evaluation of $M Y C$ and chromosome 8 copy number in breast carcinoma by interphase cytogenetics. Genes Chrom Cancer 18: 1-7

Wagner U, Bubendorf L, Gasser TC, Moch H, Görög J-P, Richter J, Mihatsch MJ, Waldman FM, Sauter G (1997) Chromosome 8p deletions are associated with invasive tumor growth in urinary bladder cancer. $A m J$ Pathol 151: $753-759$

Watters AD, Ballantyne SA, Going JJ, Grigor KM, Cooke TG, Bartlett JMS (2000) Aneusomy of chromosomes $7 \& 17$ predicts recurrence of transitional cell carcinoma of the urinary bladder. Br J Urol 84: 1-8

Watters AD, Going JJ, Grigor KM, Bartlett JMS (2002) Progression to detrusor-muscle invasion in bladder carcinoma is associated with polysomy of chromosomes 1 and 8 in recurrent pTa/pT1 tumours. Eur J Cancer 38: 1593-1599

Watters AD, Stacey MW, Going JJ, Grigor KM, Cooke TG, Sim E, Bartlett JMS (2001) Genetic aberrations of NAT2 and chromosome 8; their association with progression in transitional cell carcinoma of the urinary bladder. Urologia Internationalis 67: 235-240

Witjes JA, Kiemeney LALM, Wheeless LL, Grossman HB (2000) The value of histopathological prognostic factors in superficial bladder cancer; do we need more? Urol Oncol 5: $185-190$ 